\title{
Centralized vs Decentralized Contests
}

\author{
Carmen Beviá \\ Luis C. Corchón \\ Universidad de Alicante Universidad Carlos III de Madrid
}

October 1st, 2015

\begin{abstract}
We compare two contest. Decentralized in which there are several independent contests with non overlapping contestants and Centralized in which all contestants fight for a unique prize which is the sum of all prizes in the small contests. We study the relationship between payoffs and efforts between these two contests.
\end{abstract}

Keywords: Centralization, Decentralization, Contests

JEL classification: C72; D72; D74.

Corresponding author: Carmen Beviá. e-mail: Carmen.bevia@gmail.com

Telephone: +34965903614 


\section{Introduction}

Is it better to gather all rent-seeking activities in one place, say Washington D.C. or Brussels, available to all citizens, rather than having them scattered all over US/EU and available only to the local people? Shall research funds for, say, economics be allocated in a single large contest available to all or shall they be allocated in several small contests only available to the local people?

These kind of questions arise again and again and they involve issues of efficiency and fairness. In this note we concentrate on an important aspect of the problem namely equilibrium payoffs and efforts spent by the contestants. Effort is sometimes socially valuable, such as when it is a proxy of the quality of the job to be done by the contest winner, or is sometimes a waste from the social welfare perspective, like rent seeking efforts aiming at a monopoly franchise. The effect on effort of passing from a large contest to a several small ones is not obvious. On the one hand the small contest has less competitors so individual efforts must increase. But on the other hand the prize is now smaller which calls for less effort.

In this note, we characterize the relationship of efforts in decentralized and in centralized contests assuming the Contest Success Function (CSF) proposed by Beviá and Corchón (2015) which generalizes Tullock CSF. We also find necessary and sufficient conditions for the contestants or the contest organizer to prefer centralized or decentralized contests.

The only paper dealing with this problem is by Wärneryd (2001). He assumes a generalized Tullock CSF and identical agents. Only our result on aggregate effort (Proposition 2) is comparable to the results obtained by him, see footnote 4 .

\section{The model}

In a contest, $m$ agents called contestants spend efforts (bids) denoted by $G_{i}$ in order to win a prize of value $V_{i}$. We consider two type of contests.

- Decentralized (D) $k$ independent identical contests with $n$ contestants each (thus $m=n$ ) and a prize valued as $V_{i}, i=1,2, \ldots, n$.

- Centralized (C) A single contest which is the aggregation of $k$ identical contests. There are $k n$ agents (thus $m=k n$ ) and a single prize valued as $k V_{i}, i=1,2, \ldots, k n .{ }^{1}$

\footnotetext{
${ }^{1}$ We assume that the value of the prize in $\mathrm{C}$ is just the sum of the $k$ prizes in $\mathrm{D}$.
} 
A Contest Success Function (CSF) maps efforts of the agents into the probability that they will obtain the prize (or her share of the prize). Let $G=\left(G_{1}, \ldots, G_{m}\right)$. In a previous paper we introduced the idea of a notional CSF which maps $G$ into real numbers (Beviá and Corchón (2015)). We proposed the following notional CSF:

$$
\begin{aligned}
f_{i}(G) & =\alpha+\beta \frac{G_{i}-s \frac{\sum_{j \neq i} G_{j}}{m-1}}{\sum_{j=1}^{n} G_{j}}, i \in\{1, . ., m\} \text { if } \sum_{j=1}^{n} G_{j} \neq 0, \alpha \in[0,1], \beta \geq 0 . \\
f_{i}(G) & =\frac{1}{m} \text { if } \sum_{j=1}^{m} G_{j}=0, i \in\{1, . ., m\} .
\end{aligned}
$$

This (notional) CSF mixes proportional CSF (Tullock (1980)) and (relative) difference CSF (Hirshleifer (1989), Baik (1998) and Che and Gale (2000)). To convert this notional CSF into a CSF we first need that $\sum_{j=1}^{m} f_{i}(G)=1$. This is accomplished by the following condition:

$$
1=m \alpha+\beta(1-s)
$$

When $s=\alpha=0, \beta=1$ we have the Tullock CSF and $f(\cdot)$ is non negative. When $s \neq 0$ or $\alpha \neq 0$ non negativity is achieved when $m=2$ by introducing max min operators as in Che and Gale (2000) or for general $m$ by introducing a rationing rule which mimics the working of the CSF, see Beviá and Corchón (2015) for details. We show that in equilibrium there is no rationing so we leave the details of the rationing scheme to the interested reader. Let $h(\cdot)$ the CSF derived from (2.1) and (2.2) by taking into account that the range of such a function must yield probabilities. Consider a game in which strategies are expenses and payoff functions are

$$
\pi_{i}=h_{i}(G) V_{i}-G_{i}
$$

Let $Y_{i} \equiv V_{i} \sum_{j=1}^{m} \frac{1}{V_{j}}$. To simplify the presentation, we focus on Nash equilibria in pure strategies in which all players exert a positive effort, which is guaranteed if:

$$
Y_{i}>m-1, i \in\{1, . ., m\}
$$

which holds in the symmetric case where all valuations are identical and thus $Y_{i}=m$ for all $i$ and when $m=2 .^{2}$ Suppose a $\mathrm{D}$ contest with values $\left(Y_{1}, Y_{2}, \ldots Y_{n}\right)$. Then the corresponding values in the $\mathrm{D}$ contest are $\left(k Y_{1}, k Y_{2}, \ldots k Y_{n}\right)$. Thus, in a $\mathrm{D}$ and $\mathrm{C}$ contest $(2.5)$ reads

$$
Y_{i}>n-1, i \in\{1, . ., n\},
$$

\footnotetext{
${ }^{2}$ An identical assumption guarantees that when the CSF is of the Tullock type, all players are active in equilibrium, see Franke et al. (2013), Theorem 2.2.
} 


$$
Y_{i}>\frac{k n-1}{k}
$$

Note that (2.7) implies (2.6) so we will only use the former.

To prove the existence of a Nash Equilibrium, we need the following assumption.

$$
Y_{i}(\alpha+\beta) \geq \beta(m-1+s)\left(2-\frac{m-1}{Y_{i}}\right), i \in\{1, . ., m\} .
$$

When all players have identical valuations, (2.8) is $m \geq \beta(m-1+s)$. In Beviá and Corchón (2015) we prove that a sufficient condition for (2.8) is $\alpha+\beta \leq 1$. In the Tullock case (2.8) also holds. Taking into account $(2.3),(2.8)$ is

$$
Y_{i} \geq \beta(m-1+s)\left(m\left(2-\frac{m-1}{Y_{i}}\right)-Y_{i}\right), i \in\{1, . ., m\}
$$

In Beviá and Corchón (2015) we proved the following:

Lemma 1. Under (2.5) and (2.8) there is a Nash Equilibrium $\left(G_{i}^{*}\right)_{i=1}^{n}$ such that:

$$
\begin{aligned}
G_{i}^{*} & =\frac{\beta(m-1+s) V_{i}}{Y_{i}}\left(1-\frac{m-1}{Y_{i}}\right), i \in\{1, . ., m\}, \\
\pi_{i}^{*} & =\frac{V_{i}}{Y_{i}}\left((\alpha+\beta) Y_{i}-\beta(m-1+s)\left(2-\frac{m-1}{Y_{i}}\right)\right), m=n, k n .
\end{aligned}
$$

We proved Lemma 1 in Beviá and Corchón (2015) by constructing an auxiliary game in which payoff functions are $f_{i}(G) V_{i}-G_{i}$. This game has a unique Nash equilibrium characterized by first order conditions (FOC) of payoff maximization:

$$
\beta V_{i} \frac{\sum_{j \neq i} G_{j}\left(1+\frac{s}{m-1}\right)}{\left(\sum_{j=1}^{m} G_{j}\right)^{2}}-1=0, i \in\{1, . ., m\} .
$$

We showed that FOC hold with equality for all agents and so (2.12) yields (2.10). Thus if payoffs are non negative at (2.10) this is indeed an equilibrium. And the condition for this is (2.9).

We now study how equilibrium effort changes when we pass from a small contest with $n$ agents to a large contest with $k n$ contestants and $\beta$ and $s$ do not change. ${ }^{3}$ This is because $\beta$ and $s$ are the two parameters that are relevant to determine equilibrium effort, so we keep them constant to isolate the effect on equilibrium effort of aggregating the contests. We assume that an equilibrium exists in both the small and the large contest, which amounts to (2.8) with $m=n, k n$. Thus,

\footnotetext{
${ }^{3}$ In other words, $\alpha$ is the only parameter that changes in order to maintain $(2.3)$.
} 
Proposition 1. The effort of contestant $i$ in the $C$ contest is larger than in the $D$ contests iff

$$
k n Y_{i}-n^{2} k+1>s
$$

Proof: We have that

$$
\begin{aligned}
\frac{\beta(k n-1+s) k V_{i}}{k Y_{i}}\left(1-\frac{k n-1}{k Y_{i}}\right) & >\frac{\beta(n-1+s) V_{i}}{Y_{i}}\left(1-\frac{n-1}{Y_{i}}\right) \Leftrightarrow \\
(k n-1+s)\left(1-\frac{k n-1}{k Y_{i}}\right) & >(n-1+s)\left(1-\frac{n-1}{Y_{i}}\right) \Leftrightarrow \\
Y_{i} & >\frac{(k n-1+s)(k n-1)-(n-1+s)(k n-k)}{k n(k-1)}
\end{aligned}
$$

and the result follows.

With identical agents $Y_{i}=n$ and condition (2.13) is just $s<1$. When agents are not identical a sufficient condition for (2.13) is that $s<1$ and $i$ values the prize no less than the $Y_{i}$ that would result if all agents were identical. Next we study aggregate effort.

Proposition 2. Suppose $\beta \neq 0$. Aggregate effort is larger in the $C$ contest iff $s<1$.

Proof: From (2.10), aggregate effort is larger in the C contest iff

$$
\frac{\beta(k n-1+s)}{k \sum_{j=1}^{m} \frac{1}{V_{j}}}>\frac{\beta(n-1+s)}{\sum_{j=1}^{m} \frac{1}{V_{j}}}
$$

And the result follows.

Note that when $s=0$ (for example the Tullock contest) the C contest dominates the $\mathrm{D}$ contest (as already point out by Warneryd $(2001))^{4}$. This is because when we aggregate k decentralized contests into a single centralized contests there are two effects. The Competition Effect, namely that the $\mathrm{C}$ contest has more competitors so individual efforts must decrease, and the Prize effect, namely that the $\mathrm{C}$ contest has a larger prize which calls for more effort. When $s=0$ the prize effect dominates the competition effect. As $s$ increases, effort increases in the $\mathrm{C}$ and the $\mathrm{D}$ contests, but the impact of $s$ is larger in the $\mathrm{D}$ contest because $s$ magnifies the competition effect. We find that at $s>1$, the competitive effect dominates.

Next consider a planner (or contest organizer) who can adjust parameters $s$ and $\beta$ and wants to maximize effort. ${ }^{5}$ Thus we have that:

\footnotetext{
${ }^{4}$ In particular, Warneryd finds that with identical agents and a generalized Tullock CSF, aggregate effort is larger in $\mathrm{C}$ contests than in D contests. This is because he assumes that $f_{i}(G)=0$ when $G_{i}=0$-which implies in our case that $(1-\beta)(m-1)=s \beta$ - and that the CSF is independent of $m$, which implies $\beta=1$ and thus $s=0$.

${ }^{5}$ A planner interested in minimizing effort will choose $\beta=0$.
} 
Proposition 3. When valuations can take any value compatible with (2.5) and (2.9), the Tullock CSF maximizes aggregate effort. Any other CSF that maximizes aggregate effort yields identical effort to the Tullock CSF. If the CSF is independent of $m$ the Tullock CSF is the only CSF which maximizes aggregate effort.

Proof: Looking at $(2.10)$ the planner will make $\beta(m-1+s)$ as large as possible, compatible with the sufficient condition of equilibrium (2.9). This condition can be written as

$$
1 \geq \beta(m-1+s)\left(m\left(\frac{2}{Y_{i}}-\frac{m-1}{Y_{i}^{2}}\right)-1\right), i \in\{1, . ., m\} .
$$

So now we have to see the maximum value of the term $m\left(\frac{2}{Y_{i}}-\frac{m-1}{Y_{i}^{2}}\right)-1$ because this will serve as the bound for $\beta(m-1+s)$. The maximum value of this term occurs when $Y_{i}=m-1$ which yields

$$
m\left(\frac{2}{m-1}-\frac{1}{m-1}\right)-1=\frac{m}{m-1}-1=\frac{1}{m-1}
$$

and then the maximum value of $\beta(m-1+s)$ equals $m-1$. This happens for the Tullock CSF. Finally when $\beta(m-1+s)=m-1$ independently of $m, \beta=1$ and $s=0$.

Two remarks are in order. Firstly, the optimality of the Tullock CSF is relative to the family of CSF that arise from the notional CSF (2.1) and (2.2). This leaves out important CSF like the all pay auction. Secondly, the Proposition deals with a large domain of valuations. Therefore for smaller domains, the Tullock CSF may be not optimal. Indeed when the planner knows that valuations are identical (but unknown to her) she chooses the largest $\beta(m-1+s)$ compatible with $m \geq \beta(m-1+s)$ which is $m$. For instance $\beta=s=1$. This implies $\pi_{i}=0$ and

$$
G_{i}^{*}=\frac{V}{m}
$$

This effort is the maximum effort compatible with individual rationality, i.e. $\pi_{i} \geq 0$. Thus in this case the planner will choose the same (first best) effort in both the $\mathrm{C}$ and the $\mathrm{D}$ contest. Thus,

Proposition 4. When all valuations are identical an effort maximizing planner chooses $\beta$ and $s$ such that $\beta(m-1+s)=m$. This implements first best efforts (2.20) without knowledge of $V$ (but the planner knows that all valuations are identical). Efforts in $C$ and $D$ contests are identical.

We study the winners and losers when we pass from a D contest to a C contest. When effort is a social waste both the social planner and all contestants agree on a contest with $\beta=0$ (a pure 
lottery) in which contestants make zero effort in C and D. But when effort is socially valuable, the wishes of the social planner and the contestants may be opposite because for the contestants the less effort they make the better they are. We now show that when agents have different valuations it may be possible that the wishes of the social planner and those of a group of agents coincide.

Proposition 5. Suppose $\beta \neq 0$ and $s \leq 1$. Agent $i$ prefers the $D$ contest iff

$$
Y_{i} \in\left(n-\sqrt{\frac{1-s}{k}}, n+\sqrt{\frac{1-s}{k}}\right)
$$

If $\beta \neq 0$ and $s>1$ all contestants prefer the $C$ contest.

Proof: From (2.11) and (2.3), payoffs are larger in the D contests than in the C contest iff

$$
\frac{1+\beta(s+n-1)}{n} Y_{i}-\beta(s+n-1)\left(2-\frac{n-1}{Y_{i}}\right)>\frac{1+\beta(s+k n-1)}{n} Y_{i}-\beta(k n-1+s)\left(2-\frac{k n-1}{k Y_{i}}\right)
$$

which after some manipulations becomes

$$
Y_{i}^{2}-2 n Y_{i}-\frac{1-s}{k}+n^{2}<0
$$

The left hand side of (2.23) is a convex function with a unique minimum at $Y_{i}=n$. At this value, the left hand side of $(2.23)$ is $(s-1) / k$. Thus if $s>1(2.23)$ never holds. If $s<1$ the inequality holds between the two roots for which the left hand side of (2.23) is zero. These two roots are

$$
n \pm \sqrt{\frac{1-s}{k}}
$$

and the result follows.

Thus, if contests are very competitive $(s>1)$ all agents prefer the $\mathrm{C}$ contest. An intuition of why this is so is obtained assuming that all contestants have identical valuations. In this case individual effort is larger in the $\mathrm{C}$ contest iff $s<1$ (see Proposition 1). Since the expected revenue from the prize is the same in $\mathrm{C}$ and $\mathrm{D}$, individuals prefer the contest in which they do the less effort. When $s<1$ and all agents are identical, $Y_{i}=n$ so all contestants prefer the $\mathrm{D}$ contest. But when $s<1$ and agents have different valuations, those with low and high valuations prefer the $\mathrm{C}$ contest and agents with intermediate valuations prefer the $\mathrm{D}$ contest. This is reminiscent of "Ends against the middle" (Epple and Romano, 1996) or the "coalition of extremes" in United States Congress (Hussey 2008). The following example illustrates this phenomenon. 
Example 1. Take $n=3, k=2, s=.9$, and $\beta=.5$. Valuations are $V_{1}=\frac{28.6}{39}, V_{2}=\frac{11}{13}, V_{3}=1$ which yield $Y_{1}=2.6, Y_{2}=3.0$ and $Y_{3} \equiv 3.545$. You can check that (2.5) and (2.8) hold so an equilibrium exists. According to (2.21), agents with $Y^{\prime} s=2.6$ or 3.5455 prefer the $C$ contest and agents with $Y^{\prime} s=3$ prefer the $D$ contest. Indeed in the $D$ contests $\pi_{1}^{*}=.0955, \pi_{2}^{*}=0.1457$, $\pi_{3}^{*}=0.229$ and in the $C$ contests $\pi_{1}^{*}=0.1015, \pi_{2}^{*}=0.1434, \pi_{3}^{*}=0.239$. It is illuminating to look at equilibrium efforts: agents with lower valuations make less effort in the $C$ contests (.03200) than in the $D$ contests (.09438) so the effect on payoffs rely on pure luck: the value of the term $\alpha V_{1}$ is identical in the $C$ and the $D$ contests, .23222 ( $\alpha$ in $C$ is 0.15833 and in $D$ is 0.31667). Agents with intermediate valuations make more effort in the $C$ contest (0.1387) than in the $D$ contests (0.1363). But the probability that these agents win are 0.333 in the $D$ contests and 0.265 in the $C$ contests. Thus they pay dearly their attempt to compete with agents with larger valuations in the $C$ contest.

\section{Acknowledgements}

Thanks to Marco Serena, Alex Vazquez, Karl Wärneryd, Anil Yildizparlak and the anonymous referee for very good comments. The first author acknowledges financial support from ECO200804756 (Grupo Consolidado-C), SGR2014-515 and PROMETEO/2013/037. The second author acknowledges financial support from SEJ2005-06167/ECON.

\section{References}

[1] Baik K. H. (1998). "Difference-form contest success functions and effort level in contests". Eur J Polit Econ 14, 685-701.

[2] Beviá, C., L. Corchón (2015). "Relative difference contest success function". Theory and Decision, 78, 3, 377-398.

[3] Che Y.-K., I. Gale (2000). "Difference-form contests and the robustness of all-pay auctions". Games Econ Behav 30, 22-43.

[4] Epple, D., R. E. Romano (1996). "Ends against the middle: Determining public service provision when there are private alternatives". Journal of Public Economics, 62, 3, 297-332. 
[5] Franke J., C. Kanzow, W. Leininger, A. Schwartz (2013). "Effort maximization in asymmetric contests games with heterogeneous contestants". Economic Theory 52, 2, 589-630.

[6] Hirshleifer J. (1989). "Conflict and rent-seeking success functions: ratio vs. difference models of relative success". Public Choice 63. 101-112.

[7] Hussey, W. A. (2008). "The Coalition of Extremes: Ends against the middle in the United States Congress". U. of California, http://gradworks.umi.com/33/02/3302566.html.

[8] Tullock G. (1980). "Efficient rent-seeking". In: Buchanan JM, Tollison RD, Tullock G (eds) Towards a theory of a rent-seeking society. Texas A\&M University Press, College Station, pp $97-112$.

[9] Wärneryd, K., (2001). "Replicating Contests". Economics Letters 71, 323-327. 